Portland State University

PDXScholar

Computer Science Faculty Publications and

Presentations

Computer Science

$11-1995$

\title{
Device and Physical Data Independence for Multimedia Presentations
}

\author{
Richard Staehli \\ Jonathan Walpole \\ Portland State University \\ David Maier
}

Follow this and additional works at: https://pdxscholar.library.pdx.edu/compsci_fac

Part of the Digital Communications and Networking Commons, and the OS and Networks Commons Let us know how access to this document benefits you.

\section{Citation Details}

"Device and Physical Data Independence for Multimedia Presentations", Richard Staehli, Jonathan Walpole and David Maier, Oregon Graduate Institute Technical Report 95-020, Nov. 29, 1995.

This Technical Report is brought to you for free and open access. It has been accepted for inclusion in Computer Science Faculty Publications and Presentations by an authorized administrator of PDXScholar. Please contact us if we can make this document more accessible: pdxscholar@pdx.edu. 


\title{
Device and Physical Data Independence for Multimedia Presentations
}

\author{
Richard Staehli, Jonathan Walpole, and David Maier \\ Department of Computer Science and Engineering \\ Oregon Graduate Institute of Science \& Technology \\ Portland, Oregon, LSA \\ \{staehli,walpole,maier $\} @ c s e . o g i . e d u$
}

OGI Tech. Report 95-020

November 29, 1995

Multimedia computing promises access to any type of visual or aural medium on the desktop. But in this networked future, will every type of media be accessible from every terminal device? Current multimedia standards do not allow content that is authored for high-bandwidth workstations to scale down for low-bandwidth applications. The problem is that application requests are commonly interpreted as requests for the highest possible quality and resource overloads are handled by ad hoc methods. We can begin to solve this problem by specifying Quality of Service (QOS) requirements based on functionality rather than on content encoding and device capabilities.

The potential of distributed multimedia computing can be achieved by offering device independent and physical-data independent service interfaces. Logical data independence is also desirable, but we omit discussion of it here in the interest of brevity. Device and physical-data independence are well known principles of database system design. In multimedia systems they have the following meaning:

- The same content can be presented on devices that have different resolution and bandwidth characteristics. 


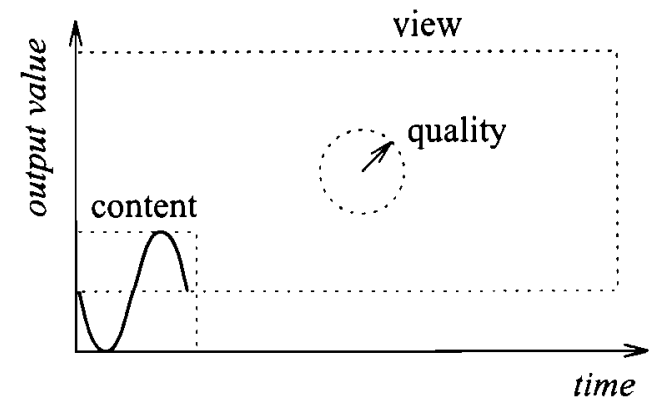

(a)

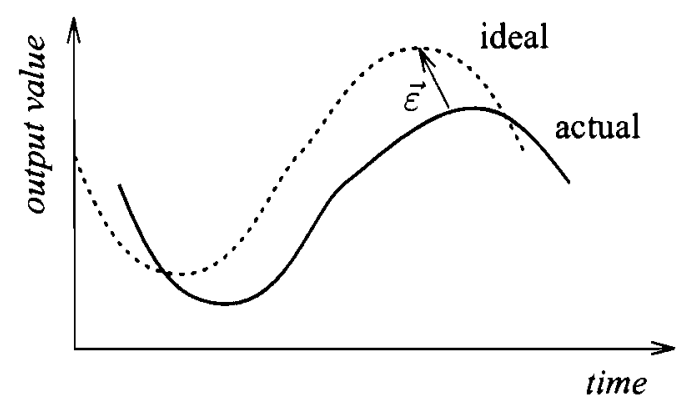

(b)

Figure 1: Content, view, and quality specify presentation QOS.

- The location and encoding of stored data should be transparent to the user.

Device independence is already supported by some content authoring standards. For example the emerging ISO MHEG standard uses virtual coordinates for content layout [3]. However, MHEG and most other authoring standards identify content with a particular encoding of data. The presentation quality for such content typically depends on the encoding, the presentation engine and the available resources. If requests for multimedia services are to have the same meaning on any platform, they should specify QOS requirements that are device and physical-data independent.

QOS specification. We propose a three-step methodology for QOS specifications: defining an ideal presentation, choosing an error interpretation, and constructing a user model.

An ideal presentation is the set of expected output values for every point in the presentation space and time. The ideal output values may vary continuously over the coordinate space of real numbers, unlike the actual output, which has finite resolution and discrete values. As a consequence, the specification of an ideal presentation is device independent, like a PostScript document. Figure l(a) illustrates the specification of an ideal presentation through a content descriptor that may be reused in many presentations and a view descriptor that specifies a particular mapping of content onto device and realtime coordinates.

An actual presentation will deviate from the ideal because of device limitations and choice of presentation algorithms and scheduling policy. Device limitations such as screen 
color depth may require dithering or some other approximation of the ideal color values. Video resolution is limited by the pixel dimensions of an output window and sample rates for both video and audio are limited by device bandwidth. The choice of compression, decompression, and rendering algorithms can introduce errors in the output values. The choice of scheduling policy affects the timing of those output values. However, just as the specification of an ideal presentation is device independent, the specification of allowable error should be independent of the mechanisms used for a presentation.

An error interpretation maps each point in an actual presentation to a point in the ideal presentation. Figure $1(\mathrm{~b})$ shows an error interpretation $\varepsilon$ for a single point in an audio presentation. The vector $\left(\varepsilon_{t}, \varepsilon_{v}\right)$ says that the value $v$ at time $t$ should have occurred at time $t+\varepsilon_{t}$ and should have had the value $v+\varepsilon_{v}$. An interpretation of error in a video presentation must also account for displacement errors in both $x$ and $y$ dimensions.

This definition allows many different error interpretations for a given pair of ideal and actual presentations. It is tempting to define a "correct" interpretation of error based on the intended correspondence of output events with content values for a particular implementation. But we want to constrain presentation outputs, not the implementation.

Finally, presentation quality requirements can be defined in terms of a user model. A user model estimates subjective presentation quality from an error interpretation. We have described a user model based on an error vector of shift, rate, jitter, and resolution components for each coordinate dimension, and a synchronization error component for the timing error between outputs [5]. These error components constitute a detailed error interpretation that can more closely model human perception. The normalized magnitude of the error vector is computed by weighting each error component according to user sensitivity. The user model "accepts" a presentation if the normalized error magnitude is within a specified limit everywhere for some error interpretation. This approach is conservative. Alternative user models might bound the average error or place other constraints on the distribution of error over an entire presentation.

QOS-Driven Presentations. Multimedia systems can provide better service if the QOS requirements of each client are known. A QOS specification can serve as a throttle to reduce resource use: requesting, for example, 24 frames/second video when a data source could supply 60 . Resource regulation is essential in a shared environment. A 


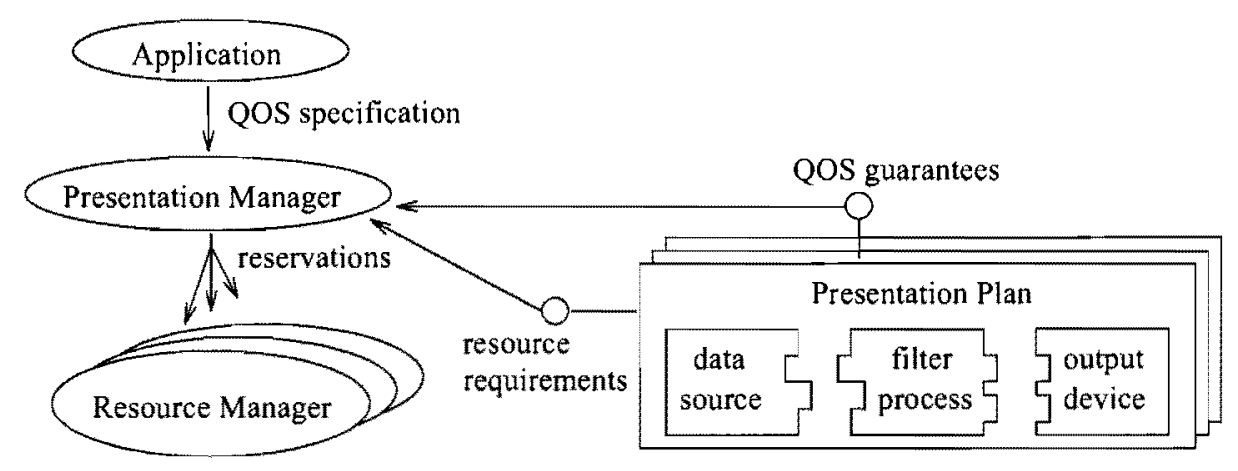

Figure 2: Presentation planning.

QOS specification can also indicate which use of resources provide the best quality for a particular presentation. For example, in a bandwidth constrained environment, an action video might be best presented at $320 \times 240$ pixels and 15 frames/second while a video of a chalkboard lecture uses the same bandwidth more effectively with $640 \times 480$ pixels and 4 frames/second.

Some systems will guarantee performance, others may only provide best-effort service. QOS specifications are needed to drive resource management decisions in both cases. Best-effort resource management still involves making decisions about how to trade one kind of resource consumption for another. This set of decisions is a planning problem that can be guided by the QOS specification. Making guarantees (which can be hard or statistical) requires an end-to-end resource reservation approach and an admission test $[2,1,4]$. Figuring out which of many different resource allocation plans is best is an optimization decision that can be guided by the QOS specification.

Figure 2 illustrates a high-level architecture for an admission test. A presentation manager receives the QOS requirements for a presentation from an application. A presentation plan is feasible if it can guarantee the QOS requirements and if the presentation manager can reserve resources for the plan. The admission test can choose to execute the feasible presentation plan with the fewest resource requirements.

Conclusion. Multimedia systems are only beginning to realize the flexibility inherent in digital computing. More work is needed to understand QOS requirements for multimedia presentations and to exploit those requirements for optimal resource man- 
agement. Device and physical-data independent QOS specifications allow applications to say what multimedia services are required without restricting how they are implemented.

\section{References}

[1] A. Campbell, G. Coulson, F. Garcia, D. Hutchison, and H. Leopold. Integrated quality of service for multimedia communications. In Proceedings IEEE INFOCOMM '93, San Francisco, USA, A pril 1993. IEEE.

[2] M.B. Jones, P.J. Leach, R.P. Draves, and J.S. Barrera, III. Support for user-centric modular real-time resource management in the Rialto operating system. In NOSSDAV 95, pages 55-66, Durham, N.H., April 1995. IEEE Communications Society.

[3] T. Meyer-Boudnik and W. Effelsberg. MHEG explained. IEEE Multimedia, 2(1):2638, Spring 1995.

[4] K. Nahrstedt and J.M. Smith. The QOS Broker. IEEE Multimedia, 2(1):53-67, Spring 1995.

[5] R. Staehli, J. Walpole, and D. Maier. Quality of service specifications for multimedia presentations. To appear in Multimedia Systems, August 1995. 